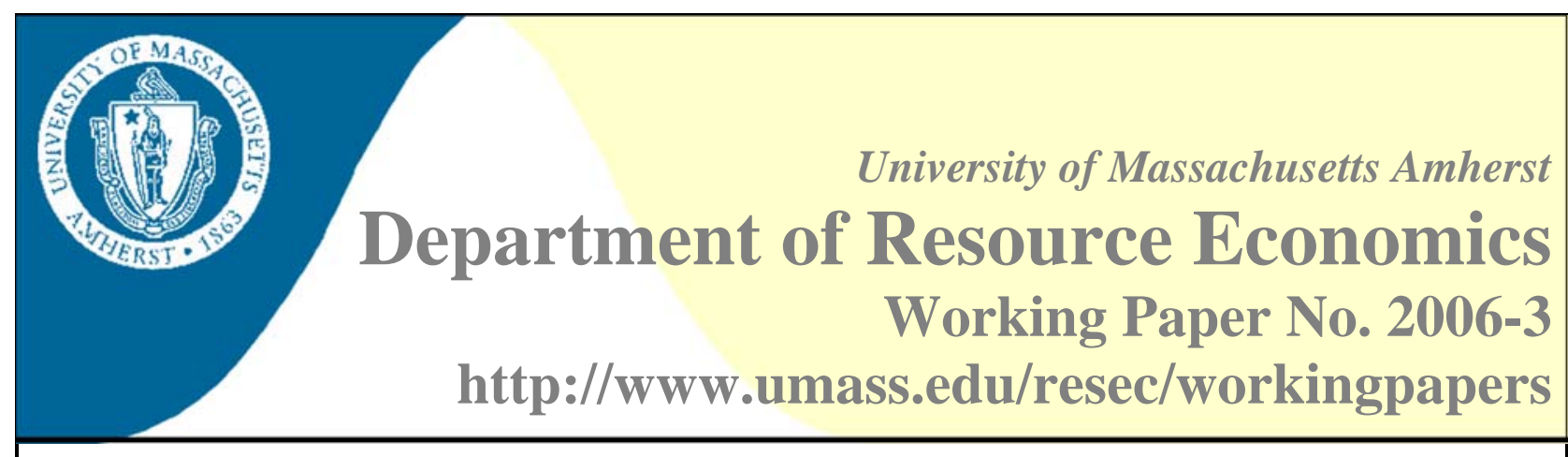

\title{
Centralized and Decentralized Management of Local Common Pool Resources in the Developing World: Experimental Evidence from Fishing Communities in Colombia
}

\author{
Maria Alejandra Velez ${ }^{1}$, James J. Murphy ${ }^{2}$, and John K. Stranlund ${ }^{3}$
}

\begin{abstract}
:
This paper uses experimental data to test for a complementary relationship between formal regulations imposed on a community to conserve a local natural resource and nonbinding verbal agreements to do the same. Our experiments were conducted in the field in three regions of Colombia. Each group of five subjects played 10 rounds of an open access common pool resource game, and 10 additional rounds under one of five institutions - communication alone, two external regulations that differed by the level of enforcement, and communication combined with each of the two regulations. Our results suggest that the hypothesis of a complementary relationship between communication and external regulation is supported for some combinations of regions and regulations, but cannot be supported in general. We therefore conclude that the determination of whether formal regulations and informal communication are complementary must be made on a community-by-community basis.
\end{abstract}

Keywords: common pool resources, experiments, institutions, communication, regulation

JEL Classification: C93, H41, Q20, Q28.

\footnotetext{
${ }^{1}$ Maria Alejandra Velez, Department of Resource Economics

University of Massachusetts, Stockbridge Hall, 80 Campus Center Way, Amherst, MA 01003

E: majavelez@gmail.com F: 413-545-5853

${ }^{2}$ James J. Murphy, Department of Resource Economics

University of Massachusetts, Stockbridge Hall, 80 Campus Center Way, Amherst, MA 01003

E: murphy@resecon.umass.edu P: 413-545-5716 F: 413-545-5853

${ }^{3}$ John K. Stranlund, Department of Resource Economics

University of Massachusetts, Stockbridge Hall, 80 Campus Center Way, Amherst, MA 01003

E: stranlund@resecon.umass.edu P: 413-545-6328 F: 413-545-5853
} 
April, 2006

\title{
Centralized and Decentralized Management of Local Common Pool Resources in the Developing World: Experimental Evidence from Fishing Communities in Colombia
}

\author{
MARIA ALEJANDRA VELEZ \\ Department of Resource Economics \\ University of Massachusetts-Amherst \\ JAMES J. MURPHY* \\ Department of Resource Economics \& \\ Center for Public Policy and Administration \\ University of Massachusetts-Amherst \\ JOHN K. STRANLUND \\ Department of Resource Economics \\ University of Massachusetts-Amherst
}

\section{Acknowledgements}

We are grateful to Maria Claudia Lopez and members of the Faculty of Environmental and Rural Studies at Javeriana University in Bogotá, Colombia who provided outstanding support for our work in the field. At each site, the experiments would not have been possible without the assistance of local community leaders that helped the research team develop credibility with local community members. We are also indebted to WWF-Colombia for coordinating the fieldwork in the Pacific Region. We received valuable comments and ideas from Sylvia Brandt, James Boyce, Juan Camilo Cardenas, and Samuel Bowles. Wendy Varner and Susanne Hale provided valuable administrative support. We assume complete responsibility for the final contents of this paper.

* Correspondence to James Murphy, Department of Resource Economics, 219A Stockbridge Hall, 80 Campus Center Way, University of Massachusetts-Amherst, Amherst, MA 01003, USA. Phone: (413)545-5716 Fax: (413)545-5853, E-mail: murphy@resecon.umass.edu. 


\title{
Centralized and Decentralized Management of Local Common Pool Resources in the Developing World: Experimental Evidence from Fishing Communities in Colombia
}

\begin{abstract}
This paper uses experimental data to test for a complementary relationship between formal regulations imposed on a community to conserve a local natural resource and nonbinding verbal agreements to do the same. Our experiments were conducted in the field in three regions of Colombia. Each group of five subjects played 10 rounds of an open access common pool resource game, and 10 additional rounds under one of five institutions - communication alone, two external regulations that differed by the level of enforcement, and communication combined with each of the two regulations. Our results suggest that the hypothesis of a complementary relationship between communication and external regulation is supported for some combinations of regions and regulations, but cannot be supported in general. We therefore conclude that the determination of whether formal regulations and informal communication are complementary must be made on a community-by-community basis.
\end{abstract}

JEL Classification: C93, H41, Q20, Q28.

Keywords: common pool resources, experiments, institutions, communication, regulation

\section{Introduction}

In this paper we report the results of a series of common pool resource experiments conducted in three regions of Colombia that depend on small-scale fishing. Our field experiments were designed to investigate whether regulations imposed on a community to conserve a local natural resource and non-binding verbal agreements within a community to do the same are complementary in the sense that a combination of formal regulations and informal community agreements lead to greater conservation of a shared local resource than either alone.

A large literature of experimental research from different disciplines has demonstrated the positive welfare effects of simply allowing subjects to communicate with each other in common pool resource settings. ${ }^{1}$ Communication can be effective because it allows participants to (1) share information about the nature of the game, its incentives and decisions that maximize group payoffs, (2) coordinate their actions and send signals about intentions, (3) express

\footnotetext{
${ }^{1}$ For recent reviews of the effects of communication in social dilemma experiments see Shankar and Pavit (2002) and Cardenas, Ahn and Ostrom (2003).
} 
displeasure about undesirable or unacceptable outcomes, (4) reduce social distance among group members, and (5) punish uncooperative behavior, for example, by agreeing not to cooperate in future periods if total group harvest exceeds some threshold.

A smaller literature has looked at the effects of external regulations-fixed quotas with some exogenous enforcement apparatus—on behavior in experimental common property games. This literature suggests that regulatory controls on the use of common pool resources may not be as effective as one would hope. Ostmann (1998) finds that external regulation and enforcement financed by experiment participants only reduces harvests by a small amount relative to a regulation-free environment. Beckenkamp and Ostmann (1999) report that middle levels of sanction lead to a reduction in the exploitation of a common property resource, but higher sanctions can cause overuse because subjects may perceive the high sanction to be unfair. Cardenas, Stranlund, and Willis (2000) found that a quota supported by weak enforcement is effective in initial rounds, but as subjects realize the weak consequences of noncompliance the effectiveness of the regulation quickly erodes. Ostrom (2000) discusses how enforcement of externally imposed rules may crowd out endogenous cooperative behavior, because it may discourage the formation of social norms to solve the dilemma, and at the same time may encourage players to cheat the system.

However, little research has been done to investigate the effects of allowing subjects to communicate under an external regulation in common pool resource experiments. We are motivated to pursue this line of inquiry because of our interest in the relationship between informal community efforts to conserve common property resources in the developing world and formal regulatory controls to do the same. Villagers in communities like those we visited typically interact and cooperate with each other on a variety of community issues. Thus, when 
examining the effects of external regulation on local natural resource use, it is unreasonable to expect that regulations would simply replace non-binding agreements among community members. Even under government regulations, community members are likely to interact with each other and develop informal norms of behavior. The question that this paper addresses is whether these informal norms and formal regulations are complementary institutions for conserving local common pool resources.

Whether communication and regulations are complementary has important implications for judging the effectiveness of government interventions in local common pool resource problems. Evaluating the performance of an intervention must be done in comparison to the performance of existing community conservation efforts, and with the recognition that community members will likely continue to pursue informal norms of behavior when the regulation is in place. Moreover, since regulatory interventions are costly, they are only justified in locales where the regulations will complement existing community efforts. ${ }^{2}$

The same processes that make communication effective in the absence of regulatory controls may also serve to complement, and be complemented by, formal regulations. For example, communication can serve as a mechanism to socialize information about the efficiency-enhancing goals of a regulation and the formal consequences of noncompliance with the regulation. Similarly, a regulation can complement cooperative community efforts if it provides a signal of efficient individual behavior that can serve as a focal point for community interactions. Moreover, group communication and the enforcement of a formal regulation can

\footnotetext{
${ }^{2}$ Bischoff (2005) is the only other study of which we are aware that combines communication and regulation in common pool experiments. Bischoff's study differs from ours in several ways, but the most important difference is that he did not examine whether communication and regulations performed better than communication alone. In fact, he finds that external regulation with communication induced a greater level of cooperation than external regulation alone. Although this result is potentially important in some settings, it does not provide the comparison between communication under a regulation to communication alone that we feel is the most relevant comparison for evaluating the performance of regulatory interventions in local common pool resource problems.
} 
provide complementary consequences for over-exploiting the resource. That is, communication can support a weak enforcement apparatus by bringing social pressure to bear on individuals to achieve more efficient outcomes, and regulatory enforcement provides an explicit sanction for noncompliance that may be necessary to support informal verbal agreements. ${ }^{3}$

On the other hand, we recognize that certain kinds of group interactions could lead to worse outcomes. It is possible that community members may implicitly transfer responsibility for resource management to the external authority. For example, group discussions may lead to a consensus that group members are in a game against the government, thereby shifting the focus away from the benefits of voluntarily coordinating actions. More specifically, communication could lead to a focus on the weak consequences of noncompliance with a regulation instead of reinforcing its welfare-enhancing objective.

We test for complementarities between formal regulations and informal non-binding communication with a series of common pool resource experiments conducted in three geographically distinct fishing areas of Colombia. Although villagers in each of these areas depend heavily on the local fishery, these areas are different along several dimensions (which we discuss briefly in Section 2). Rather than use a neutral frame, our experiments were explicitly concerned with extraction decisions from a common pool fishery. ${ }^{4}$ Thus, our experimental design avoids the problem that individuals in different communities may approach

\footnotetext{
${ }^{3}$ Baland and Platteau (1996) provide a conceptual discussion of potential complementarities between formal and informal institutions for managing common pool resources in developing countries. They suggest that such complementarities between government and user groups or communities can be exploited in co-management arrangements. Also see Bowles and Gintis (2000) and Bowles (2003).

${ }^{4}$ Within their recent taxonomy of field experiments, Harrison and List (2004) would classify our experiments as framed field experiments, because they were conducted with a population of subjects for which the phenomenon of interest (behavior in a common pool fishery) is also an important element of the subjects' experiences.
} 
a "neutral" or "decontextualized" experiment in different ways. ${ }^{5}$ Each group of five subjects first played 10 rounds of a baseline open access common pool resource game (without communication or regulation), and then 10 additional rounds under one of five institutions: face-to-face communication alone, one of two external regulations alone, and communication combined with each of the two regulations. The two external regulations consist of an individual harvest quota that was set at the level that maximizes a group’s payoff, but differ with respect to the level of enforcement. In both cases the level of enforcement was chosen to be rather weak, because this is typical of regulatory control of natural resources in the developing world. We conducted the full set of experiments in each area to determine whether the results we obtained in one region were replicable in the others.

We find no statistically significant differences in individual harvest decisions across the regions in the first-stage open access game, but significant regional variation in responses to the second-stage institutions. This suggests that the differences in responses to the second-stage institutions we observe cannot be due to regional variation in how subjects responded to the fundamental common property problem; rather, these differences must be due solely to variation in responses to the alternative institutions.

In all cases, the second stage institutions were effective in reducing harvests from the open access baseline. Thus, if we were to judge the performance of each of the regulations with respect to the open-access baseline we would conclude that they were effective in each region, although not equally so. Again, however, the appropriate comparison is between regulation combined with communication and communication alone, and a regulation can only be justified

\footnotetext{
${ }^{5}$ See the Henrich et al. (2005) experiments across 15 small-societies, and the comments by Vernon Smith, Randolph Grace and Simon Kemp (among others) in the same volume. The commentators questioned the neutral frame of these experiments because it could have been understood in different ways across the societies. Hence, the reported behavioral differences across societies could have been the result of different interpretations of the game instead of particular behavioral patterns in each society.
} 
if it complements non-binding communication. Our results suggest that the hypothesis of a complementary relationship between communication and external regulation is supported for some combinations of regions and regulations, but cannot be supported in general. We find a complementarity relationship between communication and external regulation in three out of the six possible cases. In one case, regulation and communication together led to harvest decisions that were no different from those under communication alone. In the remaining two cases, regulation combined with communication actually led to greater harvests than communication alone, suggesting that the regulation crowded-out cooperative efforts to conserve the resource.

\section{Experimental Design}

Our experiments are based on the standard problem of individual harvests from a common pool resource by $n$ identical individuals. We use a static model similar to that presented by Ostrom, Walker and Garden (1994), Falk, Fehr and Fischbacher (2002), and an earlier model developed by Cornes and Sandler (1983). Individual $i$ harvests $x_{i}$ units up to a capacity constraint $x_{i}^{\max }$. Units of harvest sell at a constant price $p$. The individual's harvest costs are $c\left(x_{i}+x_{-i}\right)+d x_{i}\left(x_{i}+x_{-i}\right)$, where $x_{-i}=\sum_{j \neq i} x_{j}$, and $c$ and $d$ are positive constants. The individual has an endowment $e_{i}$. Thus, individual payoffs are:

$$
\pi_{i}=e_{i}+p x_{i}-c\left(x_{i}+x_{-i}\right)-d x_{i}\left(x_{i}+x_{-i}\right) \text {, subject to } x_{i} \leq x_{i}^{\max } .
$$

Maximizing $\pi_{i}$ with respect to $x_{i}$, yields $i$ 's Nash best-response function:

$$
x_{i}\left(x_{-i}\right)=\min \left[\left(p-c-d x_{-i}\right) / 2 d, x_{i}^{\max }\right]
$$

provided that $p-c-d x_{-i}>0$ for all feasible $x_{-i}$. 
It is well known that pure Nash strategies result in inefficiently high harvest levels. A government authority that imposes and enforces an individual harvest quota could address this inefficiency. In this framework, inducing compliance is largely a matter of finding the correct expected penalty to reduce harvest levels to the efficient quota. However, this approach ignores other factors that may also explain individual compliance decisions. Of particular importance to us is how communication may work to support individual compliance with a formal regulation. Moreover, individuals may respond to the frame that a regulation provides; that is, that the quota provides a signal of efficient harvests, and the expected penalty signals that deviations from the quota may be punished.

Subjects were placed in groups of five and participated in a 20-period common pool resource game that was framed as a harvest decision from a shared fishery. ${ }^{6}$ Each subject received an identical payoff table that was generated from [1] with parameters $p=116.875, c=$ 17.875, $d=2.75, e_{i}=900, x_{i}^{\min }=0$ and $x_{i}^{\max }=8 .{ }^{7}$ With these values the standard symmetric Nash equilibrium is achieved when each individual chooses to harvest six units, while the group payoff-maximizing individual harvest is one unit.

Each group played a first stage with 10 rounds of a typical common pool resource game without communication or external regulation (Open Access); the second stage consisted of 10 additional rounds under one of the following institutions:

- Face-to-face communication (Communication);

\footnotetext{
${ }^{6}$ Assignment to groups was not completely random. We tried to ensure that relatives were in separate groups.

${ }^{7}$ Experiment instructions and the payoff table are available upon request <ATTACHED AS REVIEWER'S APPENDIX >. In the experiments, participants were asked to choose a harvest level between one and nine units, instead of between zero and eight units. The reason for this is that the concept of zero harvest is very difficult to explain in the field since the participants depend so critically on their use of local natural resources. The payoff table they were given was modified to account for this. However, our analysis assumes that individual harvests vary from zero and eight.
} 
- External regulation with a low penalty (Low Penalty);

- External regulation with a medium penalty (Medium Penalty);

- Face-to-face communication with a low penalty external regulation $(\mathrm{LP} / \mathrm{Com})$;

- Face-to-face communication with a medium penalty external regulation (MP/Com).

Each treatment was repeated twelve times, with four groups in each of the three regions. In the three treatments that allowed communication, participants were free to discuss anything related to the experiment prior to making their harvest decisions privately in each round. For the four treatments that involved an external regulation, an individual harvest quota of one unit (the efficient individual harvest) was imposed. To enforce the quota, each subject faced an audit probability of 10 percent. $^{8}$ If an inspection revealed that a subject's harvest exceeded one, then that person incurred a financial penalty; the results of inspections were not made public. We examine two regulations that differ only in the level of the unit penalty for discovered harvests that exceeded the quota. For the Low Penalty and LP/Com treatments, the penalty was 27 pesos per unit above the quota. We chose this penalty because the resulting expected marginal penalty is not high enough to change the pure Nash strategy equilibrium from the baseline Open Access equilibrium of six units for each individual. Nevertheless, such a regulation might serve to reduce individual harvests because of the frame the regulation places on the experiment, in particular the signal of efficient choices and that deviations from the quota will be sanctioned. For the Medium Penalty and MP/Com treatments, the unit penalty was 165 pesos. The Nash strategy equilibrium with this penalty is five units for each individual. We chose enforcement strategies that were rather weak, at least under a conventional theory of regulatory enforcement,

\footnotetext{
${ }^{8}$ In order to decide who in a group, if anyone, was inspected in a particular round, a ballot was chosen from a bag containing five ballots with the participants' numbers on them and five other blank ballots.
} 
because this is likely to be a characteristic of most regulatory controls of resource use in the developing world.

In each round, subjects were asked to choose a harvest level. After all subjects made these decisions, the monitor collected this information and announced to the group the aggregate level of harvest for that round. With this information, individuals were able to calculate their individual payoffs from the level of total harvest by the others. Individual earnings ranged between 11,220 and 22,900 pesos with an average of 15,240 pesos (about US\$6.00). ${ }^{9}$ Earnings were paid in cash at the end of each experiment. Each experiment lasted about three hours. Before each experiment began, instructions were read aloud by the monitor and several practice rounds that did not count toward final earnings were played to familiarize the participants with the experiments.

The experiments were conducted during the summer of 2004 in three distinct areas of Colombia: on the Caribbean Coast, along the Magdalena River, and on the Pacific Coast. A total of 300 individuals participated in the experiments, evenly divided among the three regions. Summary statistics of the subjects' characteristics by region are provided in Table 1 . The Magdalena and Pacific regions were roughly comparable across all five dimensions: the mean age was about 42 with almost five years of formal education. Subjects in these two regions were overwhelmingly male fishermen who had lived in the same community for more than 10 years. In the Caribbean, subjects were younger and more educated. There was also a more even gender distribution (55 percent male). Relative to the other two communities, a smaller majority of subjects lived in the same community for over 10 years and earned their living primarily from fishing.

\footnotetext{
${ }^{9}$ Daily wages in the regions where the experiments were conducted varied between 10,000 and 15,000 pesos.
} 
An important element of our design is that all treatments were conducted in each of the three regions. Our motivation for doing so was to examine whether the results we obtain in one region are replicable in the others, or if there are significant regional differences. Clearly, with only three regions, we cannot rigorously link differences in our results across the regions to differences in specific regional characteristics. We do, however, discuss how some regional characteristics may be related to the experiment outcomes we observe, so a brief description of how the regions are different is appropriate.

Participants in the Pacific region, more specifically the Ensenada de Tumaco, are members of Afro-Colombian communities, the majority of whom live in collectively owned territories. In the Ensenada de Tumaco, 94 percent of the participants report that fishing, particularly shrimp harvesting, is their main livelihood. Compared to the other two regions, government authorities charged with regulating fisheries and other natural resources have a stronger presence in this region. Colombian fisheries are regulated by INCODER (Instituto Colombiano de Desarrollo Rural), a federal level agency under the Ministry of Agricultural Affairs. INCODER enforces several regulations on the Pacific Coast, such as seasonal restrictions and the prohibition of certain methods of harvesting shrimp. In general, local fishermen in the Ensenada de Tumaco, are aware of the regulations they operate under, and there is agreement among them about the need to regulate the shrimp fishery. However, they have concerns about particular regulations, especially those that involve seasonal fishing restrictions, because of the lack of alternative employment opportunities. Community-based organizations, as well as international conservation non-governmental organizations, are also actively promoting the conservation of the natural resources of the region, in particular the mangrove forests. International conservation organizations consider this region a "hot spot" of biodiversity that they believe to be threatened. 
The participants in the town of La Dorada, Caldas, and surrounding villages are part of a mostly white and mestizo population who harvest several species of fish from the Magdalena River and the adjacent lake, Charca de Guarinocito, in the interior of the country. Eighty-seven percent of the participants reported that small-scale fishing was their main economic activity. The presence in this area of INCODER is considered to be very weak—participants describe regulatory authorities as distant, with no involvement at all with the community. Nevertheless, most of the participants are aware of seasonal restrictions on harvesting certain species. International conservation organizations are not present in this area, but a local fishermen's association has been formed to manage the local fishery. In fact, about $20 \%$ of the Magdalena participants belong to this association, which has been actively designing and enforcing their own rules for fishing in the Charca de Guarinocito.

Participants in the Caribbean region, more specifically near the city of Santa Marta, are part of a multiethnic population of whites, mestizos, African descendants, and indigenous peoples. The proportion of participants in this region who reported that fishing is their main economic activity is significantly lower than in the other two regions (69 percent). Some of the other participants are small-scale fish buyers who then re-sell their product in Santa Marta. The rest are farm workers. Generally, the participants did not know who had the authority to regulate the local fisheries. However, some methods of fishing are recognized as illegal (e.g., fishing with dynamite, minimum mesh size for nets). Fewer other fishing norms (formal or informal) are followed in this region. 


\section{Results}

To formally test for possible complementarities between formal regulations imposed on a community to conserve a local natural resource and non-binding verbal agreements to do the same, we use a linear random effects Tobit model to control for individual heterogeneity and to reflect the fact that harvest decisions were constrained between zero and eight, inclusive. We estimate individual harvest decisions as a function of a constant (interpreted as the Open Access treatment in the first stage) and dummy variables for the five second stage treatments in each of the three regions. The results are presented in Table 2. We also estimated a similar model with dummy variables for Open Access in each region and there was no statistically significant difference across the regions $(p=0.69)$. Under Open Access, average individual harvests in the three regions is 4.84 units, which is lower than the Nash equilibrium harvest (six units) but higher than efficient harvest (one unit). This observation is consistent with the results of Cardenas et al. (2000, 2002) in other Colombian communities using a similar protocol. What is particularly interesting is that the first stage Open Access results are replicated in the three regions, yet, as we will see shortly, significant regional differences emerge when we introduce the alternative institutions in stage two. This suggests that any differences in stage two results are attributable to regional interactions with the different institutions, and not to regional differences in the way in which the subject pools responded to the fundamental common pool dilemma.

As expected, the Communication treatment was effective in reducing harvests relative to Open Access in all three regions, although the effect in the Pacific region $(-0.98)$ was much lower than in the Magdalena (-2.28) and the Caribbean regions (-1.82). These regional differences are jointly significant $(p=0.00)$, although the effects of Communication in the Magdalena and the Caribbean regions are not statistically different $(p=0.18)$. This regional 
variation reveals differences in the ability of different groups to form and maintain non-binding verbal agreements to conserve the resource.

There is also noticeable regional variation in the effects of the Low Penalty. In the Caribbean, the reduction in average harvests from Open Access $(-0.69)$ is much lower than in the Pacific (-3.47) and on the Magdalena River (-1.88). These regional differences are jointly significant $(p=0.00)$. On the other hand, there is no statistically significant difference in the effects of the Medium Penalty among the regions ( $p=0.29$ ).

Why is there so much regional variation with the Low Penalty, but none with the Medium Penalty? Both treatments frame the experiments by providing a signal of the efficient individual harvest choice and by punishing deviations from this choice, but the Medium Penalty regulation has a fine for exceeding the harvest quota that is over six times that of the Low Penalty regulation. Moreover, the fine for noncompliance in the Low Penalty regulation is so low that, at least in theory, it should have no effect on harvest choices, yet in all regions there is a statistically significant reduction in harvests with this regulation. Its effectiveness, therefore, must be largely due to the regulatory frame, not the penalty. The regional variation in the effects of the Low Penalty suggests that reliance on a simple regulatory frame produces much less consistent results. In fact, in the Pacific the framing effect of the Low Penalty yielded a larger reduction in harvests than the higher monetary costs associated with the Medium Penalty ( -3.47 vs. $-2.05, p=0.00)$. In the Caribbean this relationship was reversed ( -0.69 for the Low Penalty vs. -1.61 for the Medium Penalty, $p=0.01$ ), and in the Magdalena there was no difference in the effects of the two regulations ( -1.88 for the Low Penalty vs. -2.12 for the Medium Penalty, $p=0.50$ ). Thus, while both the Low Penalty and the Medium Penalty regulations provide signals of efficient harvests and sanctions for deviating from the regulatory quota, the stronger 
monetary incentive of the Medium Penalty produced consistent reductions in average harvests across the regions while the weaker monetary incentive of the Low Penalty did not.

Overall, then, the regional variation in the responses to the second stage institutions occurs with the treatments that rely on framing effects (Low Penalty) or social pressure (Communication) to reduce harvests. In contrast, there is no regional variation in the effects of the Medium Penalty regulation, which relies instead on a significant monetary incentive for violating the regulatory quota to induce more conservative choices.

Now let us turn to our main hypothesis that communication and regulation are complementary institutions. Let us say that communication and a regulation are strong complements if combining communication with a regulation produces more conservative harvests than either communication or the regulation alone. Of the six possible combinations of regions and regulations, only two reveal a strong complementarity between communication and regulation. Note from Table 2 that in the Caribbean region the reduction of harvests in the LP/Com treatment from Open Access (-3.28) is significantly greater than the reduction achieved by both the Communication treatment $(-1.82, p=0.00)$ and the Low Penalty treatment $(-0.69, p$ $=0.00$ ). Thus, Communication and the Low Penalty regulation are strongly complementary in the Caribbean. Similarly, Communication and the Medium Penalty regulation are strong complements in the Pacific region because the harvest reductions for the $\mathrm{MP} / \mathrm{Com}$ treatment $(-2.83)$ are greater than both the Communication treatment $(-0.98, p=0.00)$ and Medium Penalty treatment $(-2.05, p=0.03)$.

However, in practical applications, the strong complementarity criterion may be too strict. The villagers that were the subjects in our experiments cooperate with each other on a large number of community issues. Thus, it is likely that a regulation to control individual harvests 
from a local fishery would be implemented in communities that already communicate with each other about the fishery, as well as other shared concerns. To judge the performance of a regulatory intervention in such a community, it is appropriate for us to ask whether a regulation complements existing community efforts, but not vice-versa. Let us say that communication and a regulation are weak complements if their combination produces more conservative harvests than communication alone. Of the six combinations of regions and regulations, there are three such cases. Obviously, if communication and a regulation are strong complements, then they are weak complements as well. Thus, we have already identified two of the three cases of weak complementarity. The other case is in the Pacific region, where the harvest reduction for the $L P / C o m$ treatment $(-2.81)$ is greater than for Communication $(-0.98, p=0.00)$.

We also observe two cases in which communication combined with a regulation actually led to worse outcomes than communication alone. When this occurs, the regulation crowds out cooperative efforts to conserve the resource. In the Caribbean region, the MP/Com treatment produced a lower reduction in average individual harvests than Communication (-1.17 vs. $-1.82, p=0.06$ ). Similarly, in the Magdalena region the $L P / C o m$ treatment yielded a lower reduction in harvests than Communication $(-1.54$ vs. $-2.28, p=0.03)$. This crowding-out does not occur with the Medium Penalty regulation in the Magdalena region. In fact, the reduction in average harvests for the $\mathrm{MP} / \mathrm{Com}(-2.63)$ is not significantly different from the harvest reduction for Communication $(-2.28, p=0.32)$.

We conclude, therefore, that the hypothesis that informal communication and formal regulatory structures are complementary is not supported generally. Of the six possible combinations of regions and regulations, we observe a strong complementarity in only two instances, and a weak complementarity in one additional case. On the other hand, there are two 
cases in which a regulation crowds out communication. In the last case, the combination of communication and a regulation does not produce a significant difference in average harvests than communication alone. Although there are likely to be regions in which regulatory control of harvests from a common pool resource complements informal community efforts, our results suggest that such a relationship will not be robust across communities and regulations.

Our results beg the question of why different regions produce different results in the same experiments—-particularly considering that outcomes under Open Access in all three regions were identical. With only three regions, we hesitate to generalize our results too broadly. Nevertheless, there may exist a mapping between the relative importance of government regulations and informal community efforts in the participants' everyday lives on the one hand and our experimental results on the other that is worth exploring with subsequent research.

Let us compare the Pacific and Magdalena regions. The subject pools in these two regions are very similar in terms of average age, years of formal education, gender composition, and livelihood (see Table 1). However, in the Pacific region the federal regulatory authority has the strongest presence of the three regions and the participants in the experiments generally agreed about the need for such regulations. In contrast, federal regulators have little involvement in the Magdalena fishery; instead, a local fishermen’s association designs and enforces informal fishing rules. Thus, the relative importance of formal regulations versus informal community efforts is significantly higher in the Pacific than in the Magdalena region. Our experiment results reveal that, in the Pacific, each of the regulatory institutions performed much better than Communication alone. In fact, both the Low Penalty and Medium Penalty regulations complemented Communication in this region. In contrast, Communication alone was very effective at reducing harvests in the Magdalena region, and was at least as effective as any of 
the regulatory treatments. Moreover, the Medium Penalty regulation did not complement Communication, and the Low Penalty regulation actually crowded out Communication. Thus, a comparison between the Pacific and Magdalena regions suggests the intriguing hypothesis that the relative importance of government regulations and informal conservation efforts in a community may be positively correlated with the likelihood that formal regulations can complement informal community efforts.

The connection between the relative importance of regulations versus community efforts and the results of our experiments is not as clear in the Caribbean. In this region there is both minimal regulatory pressure and no clear community efforts to conserve the fishery. In addition, the subject pool in the Caribbean was significantly different from those in the Pacific and the Magdalena regions. In particular, fewer of the subjects earned their living primarily through fishing and fewer lived in the community for over 10 years (see Table 1). With a less stable population that is less concentrated on fishing, it is possible that these subjects are less vested in the local fishery, resulting in a weaker mapping of the context of their everyday lives into the experimental results.

However, the fact that Communication and the Low Penalty regulation are strongly complementary in the Caribbean region, while the Medium Penalty regulation crowds out Communication, is consistent with Ostrom's (2000) hypothesis about the crowding out phenomenon. Ostrom suggests that a regulation with a modest degree of external enforcement discourages the formation of social norms and encourages players to cheat the system, whereas in situations with poorly enforced regulations, social norms evolve to support cooperation. This is exactly what we observed in the Caribbean: however, since we only observe this phenomenon in this region, we must conclude that it is not likely to be robust across communities. 


\section{Concluding Remarks}

The primary message of this work is a cautionary note concerning the performance of government interventions in small-scale resource industries in the developing world. Although each of the regulatory interventions we studied was effective at inducing more conservative harvests than under an open access scenario, this comparison is not the most relevant one for evaluating government intervention in common pools in the developing world. In most of these cases, regulatory interventions will be imposed on communities of resource users that already have informal norms about individual behavior in the commons, albeit with widely varying degrees of success. Thus, the relevant measure of the performance of a regulatory intervention is not how it performs with respect to the theoretical open access situation, but how it performs relative to existing informal conservation efforts that stem from communication and organization at the community level that may or may not continue once a regulation is in place. With regard to this comparison, we observe that regulatory interventions sometimes do more harm than good, are sometimes completely ineffective, and at other times complement existing community efforts. Since regulatory interventions are costly, they are only warranted in those communities where there is a strong likelihood that the intervention complements existing community efforts.

Identifying these communities calls for more intense study of the determinants of community responses to regulatory intervention. Geographical variation in the effectiveness of regulatory interventions could reflect existing behavioral patterns under current regulations, relationships with government authorities, and patterns of cooperation among community members to conserve a local resource (Henrich et al. 2004, Cardenas and Ostrom 2004). Clearly, further research is needed to explore how community and individual characteristics can 
explain variation in the responses to alternative institutions. Obviously, this requires visiting many more communities than we were able to. Yet, a clearer understanding of the relationships between community and individual characteristics and behavior in common pool experiments will provide valuable information about exploiting possible complementarities between community-based initiatives and external regulations, and thus help in the design of better policies to effectively and efficiently reduce overexploitation of common property resources in the developing world.

Finally, we think that our study highlights and clarifies the value of conducting framed field experiments. As we have stated several times, our broader interest is in the performance of regulatory interventions in small-scale resource industries in the developing world. Thus, rather than trying to address this issue with students in university labs, it is appropriate that we traveled to a developing country and conducted experiments that presented a common pool dilemma to individuals whose livelihood is tied to a common pool resource. The advantage of such framed field experiments is that subjects bring a context from their daily lives that could influence their experiment behavior, and that context is an important element of the question that is being addressed. The regional heterogeneity of the responses to the institutions we examined in our experiments drives our main result about the non-robustness of a complementary relationship between communication and external regulations. If we had used university students, we would have run the substantial risk of missing the heterogeneity that is so obviously important in the field.

However, the heterogeneity we observe not only highlights the value of framed field experiments, but also implies that the field itself is a heterogeneous, and often challenging, place in a way that the lab is not. Indeed, our results are a cautionary tale for anyone who 
contemplates field experiments. If we had attempted to draw conclusions about the performance of regulatory institutions in small-scale fisheries in the developing world from experiments conducted in only one region of Colombia, then the results would have been just as misleading as the results from the same experiments conducted in a lab with university students. Hence, the value of field work like ours does not come from simply designing framed experiments to examine behavior by individuals who are intimately connected to the questions of interest, although in cases like ours this is surely important. Replication in as many of the relevant settings as possible is equally important. 
Table 1: Summary Statistics of Subject Characteristics

\begin{tabular}{cccccccc}
\hline Subject Characteristics & $\boldsymbol{N}$ & Caribbean & $\boldsymbol{N}$ & Magdalena & $\boldsymbol{N}$ & Pacific \\
\hline $\begin{array}{c}\text { Mean Age (years) } \\
\text { Mean years of formal education }\end{array}$ & 97 & 6.3 & 100 & 4.7 & 93 & 4.7 \\
$\begin{array}{c}\text { Percent Male } \\
\begin{array}{c}\text { Mercent who have lived in the } \\
\text { more. }\end{array}\end{array}$ & 100 & $55 \%$ & 100 & $83 \%$ & 100 & $89 \%$ \\
$\begin{array}{c}\text { same community for 10 years or } \\
\begin{array}{c}\text { Percent for whom fishing is } \\
\text { their main activity }\end{array}\end{array}$ & 100 & $78 \%$ & 100 & $93 \%$ & 98 & $95 \%$ \\
\hline
\end{tabular}

$N$ refers to the number of responses. There were 100 participants in each of the three regions. 
Table 2: Random Effects Tobit Models of Individual Harvests

\begin{tabular}{|c|c|}
\hline Variable & Coefficient \\
\hline Constant (Open Access) & $4.84 * * *(0.08)$ \\
\hline \multicolumn{2}{|l|}{ Caribbean Region } \\
\hline Communication & $-1.82 * * *(0.25)$ \\
\hline Low Penalty & $-0.69 * * *(0.25)$ \\
\hline Low Penalty $\times$ Communication $($ LP/Com) & $-3.28 * * *(0.25)$ \\
\hline Medium Penalty & $-1.61 * * *(0.25)$ \\
\hline Medium Penalty $\times$ Communication (MP/Com) & $-1.17 * * *(0.25)$ \\
\hline \multicolumn{2}{|l|}{ Magdalena Region } \\
\hline Communication & $-2.28 * * *(0.25)$ \\
\hline Low Penalty & $-1.88 * * *(0.25)$ \\
\hline Low Penalty $\times$ Communication $($ LP/Com) & $-1.54 * * *(0.25)$ \\
\hline Medium Penalty & $-2.12 * * *(0.25)$ \\
\hline Medium Penalty $\times$ Communication $(\mathrm{MP} / \mathrm{Com})$ & $-2.63 * * *(0.25)$ \\
\hline \multicolumn{2}{|l|}{ Pacific Region } \\
\hline Communication & $-0.98 * * *(0.25)$ \\
\hline Low Penalty & $-3.47 * * *(0.25)$ \\
\hline Low Penalty $\times$ Communication $($ LP/Com $)$ & $-2.81 * * *(0.25)$ \\
\hline Medium Penalty & $-2.05 * * *(0.25)$ \\
\hline Medium Penalty $\times$ Communication $($ MP/Com) & $-2.83 * * *(0.25)$ \\
\hline$N$ & 6000 \\
\hline Prob $>\chi^{2}$ & 0.00 \\
\hline
\end{tabular}

The dependent variable is the individual's harvest and the constant is interpreted as average harvests under Open Access. Asterisks reflect $p \leq 0.01$. Standard errors (reported in parenthesis) are the same for the dummy variables for the five second stage treatments in each of the three regions because there are equal numbers of observations in each of these treatments. 


\section{References}

Baland, Jean-Marie and Jean Philippe Platteau. 1996. Halting Degradation of Natural Resources. Is there a Role for Rural Communities? Food and Agriculture Organization of the United Nations and Clarendon Press, Oxford.

Beckenkamp, Martin and Axel Ostmann. 1999. "Missing the Target? Sanctioning as an Ambiguous Structural Solution.” In Resolving Social Dilemmas. Margaret Foddy, Michael Smithson, Sherry Scheneider and Michael Hogg (Eds.), Psychology Press, Philadelphia.

Bischoff, Ivo. 2005. "Institutional Choice versus Communication in Social Dilemmas: An Experimental Approach.” Forthcoming in Journal of Economic Behavior and Organization. doi:10.1016/j.jebo.2005.01.003

Bowles, Samuel and Herbert Gintis. 2002. "Social Capital and Community Governance.” Economic Journal 112 (483), 419-436.

Bowles, Samuel. 2003. Microeconomics: Behavior, Institutions and Evolution. Princeton University Press, Princeton.

Cardenas, Juan Camilo; John K. Stranlund and Cleve E. Willis. 2000. “Local Environmental Control and Institutional Crowding Out.” World Development 28(10), 1719-1733.

Cardenas, Juan Camilo; John K. Stranlund and Cleve E. Willis. 2002. "Economic Inequality and Burden-Sharing in the Provision of Local Environmental Quality.” Ecological Economics 40(3), 379-395.

Cardenas, Juan Camilo; T.K. Ahn and Elinor Ostrom. 2003. "Communication and Cooperation in a Common Pool Resource Dilemma: A Field Experiment.” Forthcoming in Advances in Understanding Strategic Behavior: Game Theory, Experiments and Bounded Rationality: Essays in Honor of Werner Guth. Steffen Huck (Ed.), Palgrave, New York.

Cornes, Richard and Todd Sandler. 1983. “On Commons and Tragedies.” The American Economic Review 73(4), 787-792.

Falk, Armin; Ernst Fehr and Urs Fischbacher. 2002. "Appropriating the Commons - A Theoretical Explanation.” In The Drama of the Commons. Thomas Dietz, Nives Dolšak, Elinor Ostrom, Paul Stern, Susan Stonich, and Elke Weber (Eds.), National Academy Press, Washington, DC.

Harrison, Glen W. and John List (2004). “Field Experiments.” Journal of Economic Literature 52, 1009-1055.

Henrich, Joseph; Robert Boyd, Samuel Bowles, Colin Camerer, Ernst Fehr and Herbert Gintis. 2004. Foundations of Human Sociality. Economic Experiments and Ethnographic Evidence from Fifteen Small-Scale Societies. Oxford University Press, Oxford.

Henrich , Joseph; Robert Boyd; Samuel Bowles; Colin Camerer; Ernst Fehr;Herbert Gintis; Richard McElreath; Michael Alvard; Abigail Barr; Jean Ensminger; Natalie Smith Henrich; Kim Hill; Francisco Gil-White; Michael Gurven; Frank W. Marlowe; John Q. Patton and David Tracer. 2005. "Economic Man in Cross-Cultural Perspective: Behavioral Experiments in 15 Small-Scale Societies.” Behavioral and Brain Sciences 28, 795-855.

Ostmann, Axel.1998. “External Control May Destroy the Commons.” Rationality and Society 10(1), 103-122. 
Ostrom, Elinor, James Walker and Roy Gardener. 1994. Rules, Games, and Common-Pool Resources. Ann Arbor: University of Michigan Press.

Ostrom, Elinor. 2000. "Collective Action and the Evolution of Social Norms.” Journal of Economic Perspectives 14(3), 137-158.

Shankar, Anisha and Charles Pavitt. 2002. "Resource and Public Goods Dilemmas: A New Issue for Communication Research.” The Review of Communication 2(3), 251-272. 


\section{Reviewer's Appendix: Experiment Instructions ${ }^{10}$}

\section{Stage 1: Open Access Treatment (Open Access)}

Before we begin, we want to thank you all for accepting this invitation and participating in this exercise. The objective of this exercise is to understand how people make decisions related to the use of a shared natural resource. All the decisions you make, as well as all the other information you will provide us, will remain confidential. We will not divulge your individual decisions to any other member of the community, nor to any other person.

\section{Introduction}

The exercise in which you are going to participate can be different from other exercises in which members of your community might have participated in the past, therefore, any comment that you might have heard about the exercise does not necessarily apply to the version in which you will participate.

This exercise is similar to a situation in which a group of people have to make decisions on how to use a shared natural resource. For example, a forest, a drinking water source, or a fishing area. In this experiment, the resource will be referred as a fishery.

You have been selected to participate in a group of 5 people. Today, there are 3 groups participating at the same time. However, each group is independent and the decisions of the other groups do not affect the decisions of your group. Each group will be differentiated by the color of the sheets used during the exercise.

In this exercise you will earn money depending on your decisions and the decisions of the other members of your group. The reason why we use money in this exercise is to represent real life situations in which your economic decisions will bring yourself monetary consequences. You will play several rounds equivalent, for example, to periods such as years, months, or fishing seasons.

In each round, you will earn a number of points that will be equivalent to a number of pesos. At the end of the exercise, we will sum the total number of pesos earned in all the rounds, we will round the total earned, and we will personally hand that to you in cash.

We will now explain how to participate in the exercise. Please pay a lot of attention to the instructions. If you understand the instructions, you will be able to make better decisions in the exercise. Please, remain seated and do not speak with other participants. If you have a question, raise you hand. The assistant will answer your question in private.

\section{Earnings Table}

We will now hand out the EARNINGS TABLE which contains all the information you will need to make your decisions in this exercise.

\footnotetext{
${ }^{10}$ Thanks to Juan Camilo Osorio for translating the instructions from Spanish to English.
} 
All participants have the same EARNINGS TABLE that you do. The numbers in the table are points equivalent to the pesos you can earn in each round, depending on both what you decide to extract and the decisions made by others in your group.

In each round you have to decide how many units of the resource you will extract. We will call your decision "MY LEVEL OF EXTRACTION.” These units correspond to the columns 1 to 9 in the EARNING TABLE. In this exercise, each participant can extract a maximum of 9 units, and a minimum of 1 .

In the EARNINGS TABLE, the decisions of the other members of your group correspond to the column "LEVEL OF EXTRACTION OF OTHERS", which will be a number between 4 and 36. This number is the sum of the units extracted by the other members of the group. In other words, "LEVEL OF EXTRACTION OF OTHERS" is equal to: the total extraction of the whole group, minus the amount you extracted. When you make your decision, you will not know the decisions made by the other members of your group.

Once all participants hand in their decisions, we will sum all the levels of extraction and will announce the group's TOTAL LEVEL OF EXTRACTION. With this information you will be able to calculate the "LEVEL OF EXTRACTION OF OTHERS," which is equal to the “TOTAL LEVEL OF EXTRACTION” minus "MY LEVEL OF EXTRACTION”.

Let's see some examples so that you can understand how to use the EARNINGS TABLE.

Imagine you decide that "MY LEVEL OF EXTRACTION" is 4 units, and that the other members of the group extract 4 units each. We will announce that the TOTAL level of extraction is 20 units. Since you decided to extract 4, you can calculate the "LEVEL OF EXTRACTION OF OTHERS," which is equal to the "TOTAL LEVEL OF EXTRACTION" minus "MY LEVEL OF EXTRACTION". In this case, the "LEVEL OF EXTRACTION OF OTHERS" is $20-4=16$ units. Thus, as seen in the table, your earnings will be 859 .

In the previous example all the members of the group picked the same level of extraction. However, each person can pick a different number. For example, if you choose 4 and the other members of the group extract 2, 3, 7 and 8, we will announce that the TOTAL level of extraction is 24. Given the fact that you decided to extract 4, the "LEVEL OF EXTRACTION OF OTHERS" will be 20. In other words, the "TOTAL LEVEL OF EXTRACTION (24) minus "MY LEVEL OF EXTRACTION" (4). In this case, as seen on the table, your earnings will be 754.

The EARNINGS TABLE has an additional table called “Average of others". This column indicates you the average decision of your group for a determined level. For example, if the others extract 8 , this means that the average amount extracted per person is 2. Instead, if the others extract 20 , the average amount extracted per person is 5 .

Take a few seconds to look at the EARNINGS TABLE and understand how it works. If you have any questions, please raise your hand and someone will come to you. 


\section{Decision Card}

I will now explain how you will inform us in each round your level of extraction. In each round you will receive a "decision card". The decision cards are these small pieces of paper.

\begin{tabular}{|c|c|}
\hline \multicolumn{2}{|c|}{ DECISION CARD } \\
\hline Participant Number: & \\
\hline Round Number: & \\
\hline $\begin{array}{c}\text { My level of extrac1tion: } \\
\text { (a number between 1 - 9): }\end{array}$ & \\
\hline $\begin{array}{c}\text { How much do you think } \\
\text { others will extract? } \\
\text { (a number between 4-36): }\end{array}$ & \\
\hline
\end{tabular}

In each round you will have to write:

- The number of the round, which will be announced by us.

-“MY LEVEL OF EXTRACTION”, in other words, how many units will you extract, which in this case will be a number between 1 and 9 .

-You also have to write what you think the other members of your group will extract.

This is the sum of the levels of extraction that you think the other 4 members of your group will extract. This sum is a number between 4 and 36. Remember that when you make your decision you do not know what the others are choosing. However, we want to know how much you think the others will extract. For example, if you think that two people will choose 3 and the other two 5 , then, what you think the others will extract is $16(3+3+5+5)$.

What you write on the level of extraction of others will not affect your earnings, either if it is equal or different to what actually happened. However, we are interested to know what you are thinking about the level of extraction of the others when you make your choice.

After all the members of your group have made their decisions, we will pick up the 5 participants' cards and calculate the groups' TOTAL level of extraction. Once we announce the total extraction of the group you will be able to calculate the true "LEVEL OF EXTRACTION OF OTHERS.” With this information and your level of extraction, you will be able to calculate how much you earned by looking at the EARNINGS TABLE.

It is very important that you remember that your decisions are private and that you can not show them to the other members of the group. We will only announce the TOTAL level of extraction. 


\section{Calculation sheet}

Each one of you will receive a calculation sheet with which you record your decisions and earnings. Please write your participant number in the calculation sheet. This is the same number that is written in the decision cards.

Let's see how to use the calculation sheet by looking at an example. Suppose you decided to extract 4 units. In consequence, you have to write 4 under column A of the calculation sheet, as shown in the example. You should also write this number in "MY LEVEL OF EXTRACTION" in the Decision Card .You are writing your decision in two places, in the Decision Card, which you will hand in back to us, and in the calculations sheet. Please, check that you have written the same number in the two sheets before you hand in the decision card.

After all the members of the group have finished taking their decisions, we will pick up the cards of the 5 participants and calculate the groups' TOTAL level of extraction.

Suppose the "TOTAL LEVEL OF EXTRACTION" is 20 units. You should write 20 in the column $\mathrm{B}$ in the calculations sheet. In order to calculate accurately the "LEVEL OF EXTRACTION OF OTHERS," you should subtract Column A ("MY LEVEL OF EXTRACTION") from Column B (“TOTAL LEVEL OF EXTRACTION”) You should write the result in Column $C$ ("LEVEL OF EXTRACTION OF OTHERS") In our example, the “LEVEL OF EXTRACTION OF OTHERS” is $16(20-4$.

In order to calculate your earnings, you should use the EARNINGS TABLE. In this case, given that "MY LEVEL OF EXTRACTION" is 4 and the "LEVEL OF EXTRACTION OF OTHERS" is 16 , then your earnings will be 859 . This is the information you should write in column D.

\section{Practice rounds}

Before we begin the exercise we will do some practice rounds. The decisions that you take in these practice rounds would not affect your earnings today.

The first practice round will be done altogether. First, write the number of the round in the decision card, in this case (P) of practice. After that, looking at the EARNINGS TABLE suppose that each one of you picked 5. Write this in the decision card and in Column A of the earnings sheet. You should also write in the decision card what you think the other members of your group will extract. In this case, it is 20, because we know that all of them picked 5. Remember, when we begin the real exercise, you will not know the exact number of extraction of the other members while you will be picking your level of extraction. In the next rounds you will write what you think the others will extract.

Given that all the members of the group picked 5 in this example, the total level of extraction for the group is 25. Each one should write now 25 under Column B ("TOTAL LEVEL OF EXTRACTION”) in the calculations sheet.

Now subtract "MY LEVEL OF EXTRACTION" (5) from the "TOTAL LEVEL OF EXTRACTION” (25). In other words, column B minus Column A. This operation is equal to 20. This number is the true "LEVEL OF EXTRACTION OF OTHERS", which you should write in 
Column C. Using the number in Column A, "MY LEVEL OF EXTRACTION," and the number under column C, the "LEVEL OF EXTRACTION OF OTHERS", you should use the earnings table to determine your earnings for this round. In this case, your earnings will be 790 . Write your earnings in column D.

We did this example and the previous one supposing that everyone picked the same level of extraction. However, when you make your decision, you may choose the level of extraction that you want by looking at the EARNINGS TABLE. Are there any questions?

Let's continue with the next practice round. First, write down the round's name in the decision card, in this case (P) of practice. Now, each one of you has to decide your level of extraction using the EARNINGS TABLE. Write it down in the decision card and in Column $\mathrm{A}$ in the calculations sheet. Before you hand in the decision card, check that the number in column A is equal to the one you wrote in "MY LEVEL OF EXTRACTION" in the decision card. You should also write in the decision card the level of extraction that you believe the other members of the group will extract. 


\section{Stage 2 - Communication Treatment (Communication)}

We now will begin the second part of this exercise. This part is very similar to the previous one, with the difference that in the next rounds you will be able to communicate with the other members of your group.

At the beginning of each round, before you decide how much you will extract, you will have 5 minutes to discuss the exercise with the other members of your group. During these 5 minutes, you will be able to discuss with them anything related with the exercise. Before the discussion round begins, turn your calculations sheet upside down and leave it on the table. After 5 minutes, the discussion will come to an end and you will return to your seat to decide privately what your level of extraction decision will be.

\section{Stage 2 - Mid Penalty Treatment (Medium Penalty)}

(These are the instructions for the medium penalty of 165. The instructions for the low penalty treatment are identical, except that the penalty for each additional unit extracted is 27).

We will now begin the second part of this exercise. This part is very similar to the previous one, with the difference that in the next rounds we will establish a regulation. The objective of this regulation is to make all participants of your group extract $\mathbf{2}$ units.

In order to promote that people do not extract more than 2 units of the resource, we are going to impose a penalty of 165 points for each additional unit extracted. The table that we will be handing out summarizes the total penalties that result from each level of extraction.

(Hand out the tables and explain them)

\section{Penalty Table}

\begin{tabular}{|c|c|c|c|c|c|c|c|c|c|}
\hline $\begin{array}{c}\text { My level of } \\
\text { extraction }\end{array}$ & $\mathbf{1}$ & $\mathbf{2}$ & $\mathbf{3}$ & $\mathbf{4}$ & $\mathbf{5}$ & $\mathbf{6}$ & $\mathbf{7}$ & $\mathbf{8}$ & $\mathbf{9}$ \\
\hline Penalty & 0 & 0 & 165 & 330 & 495 & 660 & 825 & 990 & 1155 \\
\hline
\end{tabular}

You will have to pay the penalty only if you are inspected when extracting more than 2 units of the resource. In other words, you can extract more than 2 units, but if you are inspected, you will have to pay the penalty. However, it will be very difficult to inspect the decisions of all the members of the group.

In consequence, once each person decides their level of extraction (a number between 1 and 9) and has handed in the decision card, we will randomly pick who will be inspected.

In order to decide who will be inspected, we will take a ballot from a bag containing 5 ballots with the participants' numbers on them, and 5 other blank ballots. 


\section{(Show the ballots)}

This implies that for each round you have ONE chance in 10 of being inspected. If your number is selected and you extracted more than 2 units of the resource, then you will have to pay the penalty for every additional unit. Nobody else will ever know the result of such inspection. If a blank ballot is selected, no one will be inspected.

\section{(Practice picking the ballot)}

In each round we will only take one ballot. The selected ballot will be returned to the bag. This means that the result of the inspection in this round will not affect the result of the next one. In this way, someone can be inspected more than one time during the exercise. It is also possible that you never get inspected.

\section{Let's make some examples:}

For example, if you extract 5 units of the resource, your level of extraction is 3 units greater than the permitted level of 2 units.

If your number is randomly selected from the bag, then you will be inspected. Since you extracted 5 units, the penalty will be 495 , as can be seen in the penalty table ( $5-2=3$ and $3 * 165=495)$. In consequence, we will have to subtract 495 from your earnings.

If a blank ballot is selected nobody will be inspected and we will follow on to the next round. If you are not inspected, you will not have to pay any penalty.

Another example:

If you extract 2 units, your level of extraction is equal to the level permitted. If your number is randomly selected from the bag, you will be inspected. Given the fact that your level of extraction is equal to the permitted level, then you will not have to pay the penalty. If a blank ballot is selected, nobody will be inspected and we will pass to the next round. If you are not inspected, you will not have to pay the penalty.

\section{Calculation sheet}

The calculation sheet for these rounds is very similar to the one used in the previous rounds, but includes some changes.

Before we begin, please write your participant's number in the new calculation sheet.

Columns A, B and C are used as in previous rounds. In column D you must write your earnings before you know if you will be inspected. Now, there are two additional columns. In column E you must write whether you were inspected or not in each round. Write YES or NO. In column $\mathrm{F}$ we will write the total value of the penalty you will have to pay if you are inspected. If you were not inspected, please write 0 in this column. In the last column, column G, you can write your earnings after the inspection. Earnings after the inspection are calculated subtracting the value of the penalty (column F) from the earnings before the inspection (column D). If you were not inspected, or your penalty is 0 , your earnings (column $G$ ) will be exact to what was written in column D (My earnings before the inspection). 
Let's see some examples:

Suppose that your level of extraction was 5 units and the total level of extraction was 17 units. Write this information in the corresponding columns as in the previous rounds. In this case, level of extraction of the others is 12 (column C) and your earnings will be 1021, before the inspection.

If your number is randomly selected from the bag, then you will be inspected. Write "YES" in column E. Since you extracted 5 units, the penalty will be 495, as you can see in the penalty table (5-2 =3 and 3*165=495.) Write 495 in column F (total penalty value.) In consequence, your total earnings for this round will be 1021 (column D) - 495 (column F) $=526$. Write 526 in the last column (My earnings after the inspection, column G.)

If a blank ballot is selected and nobody is inspected we will pass the following round. You will not have to pay any penalty. Write NO in column E, 0 in column F (penalty value) and write your earnings before the inspection (column D) in column $\mathrm{G}$ (earnings after the inspection.)

\section{Stage 2 - Mid Penalty and Communication Treatment (MP/Com)}

These instructions are identical to the medium penalty treatment explained above, except that after the practice rounds are over, participants are informed that communication is allowed as in the communication treatment. The instructions for the low penalty and communication treatment are identical, except that the penalty for each additional unit extracted is 27. 
EARNINGS TABLE

\begin{tabular}{|c|c|c|c|c|c|c|c|c|c|c|}
\hline & \multicolumn{9}{|c|}{ My level of extraction } & \multirow[b]{2}{*}{$\begin{array}{c}\text { Average } \\
\text { of the } \\
\text { others }\end{array}$} \\
\hline $\begin{array}{c}\text { Level of } \\
\text { extraction } \\
\text { of others }\end{array}$ & 1 & 2 & 3 & 4 & 5 & 6 & 7 & 8 & 9 & \\
\hline 4 & 900 & 996 & 1087 & 1172 & 1252 & 1326 & 1395 & 1458 & 1516 & 1.0 \\
\hline 5 & 882 & 976 & 1064 & 1146 & 1223 & 1295 & 1361 & 1421 & 1476 & 1.3 \\
\hline 6 & 864 & 955 & 1040 & 1120 & 1194 & 1263 & 1326 & 1384 & 1436 & 1.5 \\
\hline 7 & 846 & 934 & 1017 & 1094 & 1165 & 1231 & 1292 & 1347 & 1396 & 1.8 \\
\hline 8 & 829 & 914 & 994 & 1068 & 1137 & 1200 & 1258 & 1310 & 1357 & 2.0 \\
\hline 9 & 811 & 893 & 970 & 1042 & 1108 & 1168 & 1223 & 1273 & 1317 & 2.3 \\
\hline 10 & 793 & 873 & 947 & 1016 & 1079 & 1137 & 1189 & 1236 & 1277 & 2.5 \\
\hline 11 & 775 & 852 & 923 & 989 & 1050 & 1105 & 1154 & 1198 & 1237 & 2.8 \\
\hline 12 & 757 & 831 & 900 & 963 & 1021 & 1073 & 1120 & 1161 & 1197 & 3.0 \\
\hline 13 & 739 & 811 & 877 & 937 & 992 & 1042 & 1086 & 1124 & 1157 & 3.3 \\
\hline 14 & 721 & 790 & 853 & 911 & 963 & 1010 & 1051 & 1087 & 1117 & 3.5 \\
\hline 15 & 703 & 769 & 830 & 885 & 934 & 978 & 1017 & 1050 & 1077 & 3.8 \\
\hline 16 & 686 & 749 & 807 & 859 & 906 & 947 & 983 & 1013 & 1038 & 4.0 \\
\hline 17 & 668 & 728 & 783 & 833 & 877 & 915 & 948 & 976 & 998 & 4.3 \\
\hline 18 & 650 & 708 & 760 & 807 & 848 & 884 & 914 & 939 & 958 & 4.5 \\
\hline 19 & 632 & 687 & 736 & 780 & 819 & 852 & 879 & 901 & 918 & 4.8 \\
\hline 20 & 614 & 666 & 713 & 754 & 790 & 820 & 845 & 864 & 878 & 5.0 \\
\hline 21 & 596 & 646 & 690 & 728 & 761 & 789 & 811 & 827 & 838 & 5.3 \\
\hline 22 & 578 & 625 & 666 & 702 & 732 & 757 & 776 & 790 & 798 & 5.5 \\
\hline 23 & 560 & 604 & 643 & 676 & 703 & 725 & 742 & 753 & 758 & 5.8 \\
\hline 24 & 543 & 584 & 620 & 650 & 675 & 694 & 708 & 716 & 719 & 6.0 \\
\hline 25 & 525 & 563 & 596 & 624 & 646 & 662 & 673 & 679 & 679 & 6.3 \\
\hline 26 & 507 & 543 & 573 & 598 & 617 & 631 & 639 & 642 & 639 & 6.5 \\
\hline 27 & 489 & 522 & 549 & 571 & 588 & 599 & 604 & 604 & 599 & 6.8 \\
\hline 28 & 471 & 501 & 526 & 545 & 559 & 567 & 570 & 567 & 559 & 7.0 \\
\hline 29 & 453 & 481 & 503 & 519 & 530 & 536 & 536 & 530 & 519 & 7.3 \\
\hline 30 & 435 & 460 & 479 & 493 & 501 & 504 & 501 & 493 & 479 & 7.5 \\
\hline 31 & 417 & 439 & 456 & 467 & 472 & 472 & 467 & 456 & 439 & 7.8 \\
\hline 32 & 400 & 419 & 433 & 441 & 444 & 441 & 433 & 419 & 400 & 8.0 \\
\hline 33 & 382 & 398 & 409 & 415 & 415 & 409 & 398 & 382 & 360 & 8.3 \\
\hline 34 & 364 & 378 & 386 & 389 & 386 & 378 & 364 & 345 & 320 & 8.5 \\
\hline 35 & 346 & 357 & 362 & 362 & 357 & 346 & 329 & 307 & 280 & 8.8 \\
\hline 36 & 328 & 336 & 339 & 336 & 328 & 314 & 295 & 270 & 240 & 9.0 \\
\hline
\end{tabular}

